\title{
Discussion on Application of Physical Theory Structure Framework in Physics Teaching and Scientific Research

\author{
Zhicong Fang
} School of Automotive and Electronic Engineering, Xichang College, Xichang, 615013,
China

Keywords: physical theory structure, symbolic structure, historical structure, extensional structure

\begin{abstract}
It is thought in this paper that physical theory structure includes symbolic structure, historical structure and extensional structure. The symbolic structure of physical theory is a transformational approach of physical theories. The historical structure of physical theory is the supplement of symbolic structure, while the extensional structure of physical theory is used for well communications between natural science and human science. The three structures mutually supplement and restrict, jointly acting on physics teaching and research. It is of great significance to discuss the effects of physical theory structure framework on physics teaching and scientific research for our engagement in physics teaching and scientific research in the future.
\end{abstract}

Since the $21^{\text {st }}$ century, educational reform is always a hot topic in China. The new curriculum reform emphasizes innovations of teaching. Bruner's structuralism teaching thought is served as the basis for China's physics teaching, and it is applicable to physics teaching of different disciplines. Therefore, this thought is of great importance for the research on application of structure in physics teaching and scientific research. Compared with other disciplines, physical theory has many characteristics. A perfect combination of physical theory, teaching theory and scientific research theory can be achieved only by deeply mastering such characteristics.

\section{Introduction to Physical Theory Structure}

According to the summary of experiences for physics teaching and scientific research, physical theory structure framework is mainly composed of symbolic structure, historical structure and extensional structure. In the next paragraphs, an analysis on the physical theory structure framework will be made from three perspectives.

(I) Symbolic Structure of Physical Theory

The symbolic structure of physical theory mainly refers to the large number of references to mathematical tools. Some physical phenomena will be interpreted by inevitably using quantitative laws which jointly constitute the symbolic structure together with speed, quality, power, energy and other concepts.

In most cases, physical concepts are defined through certain mathematic relations or even mathematical formulas. Therefore, this mathematical definition provides physics with favorable symbolic structure, while such symbolic structure also provides students with great probabilities to transform from the original knowledge structure to the future knowledge structure.

(II) Historical Structure of Physical Theory

The historical structure of physical theory means a sort of historical and non-mathematical-logic relationship due to differences of physics in structure and scientific research of basic theory. 
Mathematics can not meet needs of physical research, so physicists are required to utilize a certain scientific approach to generate certain theoretical relationship under the circumstances. Thus, it can be seen that the generation of historical structure is caused by personal knowledge structures of physicists and the current situation of theoretical research. In nature, its generation manifests as different results ${ }^{[1]}$ obtained by various combinations of a certain scientific approach.

(III) Extensional Structure of Physical Theory

The extensional structure of physical theory mainly indicates a sort of extensional relationship between physical theory and individual, and it manifests as the common \& special and objective \& individual relationship. Although physics is about descriptions on the objective nature, physics must be emerged with people as media. Thus, it should be extended to human bodies and act with people’s knowledge structures, so as to form a certain physical theory.

\section{Application of physical Theory's Symbolic Structure in Physics Teaching and Scientific Research}

(I) Symbolic structure is the basis for knowledge cohesion on class.

Physical textbook is served as the solid basis for the whole physics teaching and research. For the description on the whole textbook, the description on some theories can be used as the basis. Then, these theories are subject to mutual supplement. These theories jointly constitute a physical theory system containing all aspects of this process.

For instance, Newton's laws of motion are served as bases for the whole mechanics and all physical textbooks. Through Newton's laws of motion, we can infer energy conservation and other laws. This method through mathematical deduction proves that mathematics can be used to predict the whole physical theory ${ }^{[2]}$.

(II) Symbolic structure lays a foundation for relations of different textbooks.

Different physical theories have different basic theories. Moreover, their deduction processes are also not always the same. Thus, different observation methods and results will be generated. However, a sort of community exists in physics, and all physics teaching cases are to teach a certain "normal form”. This "normal form” can have an effect on the entire result.

Physics has different basic sequences. If only one theoretical sequence is used, students can only experience one side. For instance, it needs to take statics as the research basis when Newtonian mechanics is selected as the basis for physical textbooks. Thus, the research on several objects is transformed into the research on one object, while momentum can be used as main carrier to analyze the transfer process of the entire physical quantity in other textbooks. Through studies of this system, students can understand another side of physics. Studies in this way only change the sequence of theory instead of the generation of mutually contradictory theoretical issues. Therefore, differences produced by different systems can be used for supplementary learning, so 
as to make students' knowledge to be expanded ${ }^{[3]}$.

Thus, it can be seen that different textbooks are formed by the combination of different structural systems, while different textbooks also provide students with different sides of physics, helping students to reflect on relevant issues from many aspects. On the one hand, physical creative teaching is provided as different physical theories conform to the existence of structure. On the other hand, this different "normal form” will not lead to the generation of students' thought confusion, because symbolic structures of different physical theories are uniform, free from any effect on students' previous knowledge structure.

\section{Application of physical Theory's Historical Structure in Physics Teaching and Scientific Research}

(I) Physical scientific methods are served as the bases for theoretical foundations of education. Actually, the historical structure of physical theory provides a kind of physical scientific method. Although physical theory itself excludes any scientific method and it is only the response to objective laws, it can combine any scientific method, including the historical structure method of physical theory.

(II) Strategies for education with physical scientific method

Education with physical scientific method includes students' direct experience and physics history transfer. The both items don't exist independently, and they often represent a complementary relationship. Students can feel the teaching process through direct experience, and teachers can transfer scientific methods through physics history ${ }^{[4]}$.

\section{1、 Students' direct experience}

Many physical theories are results obtained by physicists' personal experiences in tests. For instance, Coulomb law is obtained by Coulomb through experiments according to the law of universal gravitation. In physical textbooks, the law of universal gravitation is usually put before Coulomb law, for the convenience of students to obtain Coulomb law by analogy from the law of universal gravitation, and beneficial to the development of teaching and scientific research. The teaching method from law of universal gravitation to Coulomb law is a historical structure, because we are unable to obtain the law of universal gravitation by analogy from Coulomb law.

2、 Transfer of physics history

Direct experience is by no means applicable to all physics teaching cases. In most cases, the physics history transfer method needs to be used. The process deducted from physical theory in the experiment is not very obvious and determined, so this process can be obtained through experimenters' repeated trials for many years. Although conclusion and analysis in physics are relatively simple methods easy to be understood, the entire experimental operation process is 
troublesome, and it needs to judge errors and phenomena through mature experiences and rich knowledge. For instance, an opportunity is lost due to an error while Ampere found electro-magnetic induction.

First of all, students' learning details are important parts. In most cases, students learn about physical knowledge through experiment by imitation. Thus, it requires providing some materials to be imitated in physics teaching.

Secondly, students should learn about analogy and deduction, especially the deduction of knowledge to be learnt about from mastered knowledge. According to Kepler, for instance, the sun's autorotation is caused by planets' movement towards the sun by the sun's emitting some substances to planets. Although their movement type is inconsistent with lights of the sun, there is certain correlation between both items. Thus, it just likes a certain instrument using sunlight. Kepler just grasped similarities of both items for further analogy and then drew an important conclusion. However, we should also pay attention to era while education with physical science method, and can not use historical structure. Moreover, we need to study Kepler's spirit of analogy and deduction ${ }^{[5]}$.

It is not occasional to discover physical theory. However, it is historically inherent law. Therefore, scientific methods should be put into that age for analysis in practical teaching and research, so as to really understand its nature. Although historically complex deduction process is already unnecessary in modern physics teaching and simple and direct methods are usually used, the effect of this historical structure lies on clear explanations of relations between various physical theories, so as to let students to master physical theory knowledge in a better way and increase students' interests in physics.

\section{Application of physical Theory's Extensional Structure in Physics Teaching and Scientific Research}

Source of emotional attitude value

Application of the extensional structure of physical theory in physics teaching and research is actually the application of emotional value. Emotional value comes from the extensional structure of physical theory, namely teachers or physics researchers. According to the author's experiences in scientific research on physics teaching for many years, physical emotional attitude value is mainly sourced from the following aspects:

1、 Students' behavioral process

Students' learning and scientific process will certainly produce certain emotional attitude values. In studies, this value will be associated with physical theory. Although this emotional is not a behavior, its emergence must be proceeded in a certain behavior. Thus, it can be represented. 


\section{2、 Cognitive process}

Physical theory carries a certain sense of beauty. This sense of beauty will motivate students' emotions in learning and physical research. For instance, physical theory includes plenty of natural phenomena. Students will produce the sense of beauty while accepting these physical phenomena. Their affection to physics will be stimulated. Additionally, physical theory also includes the beauty of concision. Simple words can be used to contain abundant information in physical theory. In the process of knowledge learning, students will experience this beauty of concision ${ }^{[6]}$.

The cognitive process of new knowledge is not simple teaching of theories. Instead, it is the inquiry-based learning process of physical knowledge. In this process, students should utilize and conclude several methods. Thus, it needs to correlate physics with phenomena in practical life for analysis. This process will inevitably bring about emotional attitude value. For instance, seesaw in practical life can be correlated while learning lever principle. Thus, students will experience charms of seesaw in the learning process, so as to experience miraculous physical theory.

\section{3、 Textual infection}

Due to limitations of knowledge level, not all emotional attitude values are directly experienced by students. Instead, some emotional attitude values are infected through text. Firstly, emotional attitude values can be cultivated through finding of physical law. For instance, teachers can introduce Faraday's successful finding of the law of electromagnetic induction after countless failures while taking about Faraday's doubt for Ampere's electro-magnetic induction. In this process, students will admire Faraday, so as to produce certain emotional attitude values.

Educational strategies for emotional attitude value

Physical theory has great specialty. Physical theory itself has no emotional attitude value, and it only exists between individuals. Therefore, teachers should pay attention to the combination of physical knowledge and individual and apply this emotional attitude value into the teaching process while physics teaching and scientific research. In addition, the extensional structure of physical theory provides the combination of other disciplines and physical knowledge. Physical theory can also accept emotional attitude value within a certain scope. However, emotional value can not be directly correlated with physical knowledge in practical teaching. Instead, it needs to first analyze physical knowledge and then find out emotional attitude value suitable for physics teaching materials.

1、 Drive emotional attitude value education through experiment

Students' direct experience is the main source to cultivate students' emotional attitude values, as physics teaching is mainly proceeded through physical experiment. In experiment, the experiment should be controlled within certain difficulty, so as to ensure that the experiment is performed 
within students' controllable scope. Thus, students can be made to maintain certain interests in physical theory knowledge and certain emotional attitude values.

2、Make cognitive conflicts

Teaching of physical knowledge is not a simple teaching process. Teaching in this way will make students to produce undesirable emotions. Therefore, teachers should selectively choose some physical theory phenomena, letting students to produce cognition for natural beauty, so as to produce cognition for physical formula. Thus, students' emotional attitude values can be cultivated. For instance, teacher can select some beautiful natural pictures and associate these pictures with mechanical movement while teaching mechanical movement. Thus, students can be made to understand charms of the nature while learning knowledge.

3、Associate with practical life

Compared with other disciples, physics has very strong practical value. In practical life, many inventions are made according to certain physical knowledge. Therefore, it is full of physical knowledge in our life. In teaching and research, teachers should associate physical theory knowledge with practical values, tell practical values represented in theories to their students, and accomplish the education of emotional attitude value. At the same time, such physical theory knowledge of emotional attitude value education must be closely associated with teaching programs, so as to achieve the purpose of improving students' performances.

\section{Conclusion:}

It can be seen through above analysis that the physical theory structure includes symbolic structure, historical structure and extensional structure. The symbolic structure of physical theory is a transformational approach of physical theories. The historical structure of physical theory is the supplement of symbolic structure, while the extensional structure of physical theory is used for well communications between natural science and human science. The three structures constitute three dimensions of scientific research on physics teaching, jointly acting knowledge, method and emotion on scientific research on teaching. Only proper combination between this teaching theory and physical theory knowledge can achieve the objective of teaching knowledge and overcoming difficulties.

\section{References:}

[1]Shao Ruizhen, Bruner's Curriculum Theory [J], Global Education, 2009, 09 (05): 10-11;

[2] Chen Xianglei, Characteristics of Bruner's Teaching Theory Thought and Design on PSSC Physics Course [J], Global Education, 2010, 04 (10): 81-82;

[3] Chen Changcen, Innovation to Teaching Theory and Practice - Review for Ausubel's 
“Advance Organizer” [J], Comparative Education Review, 2005, 09 (05): 19-20;

[4] Guo Yiling, Several Opinions on Application of Experimental Physics History in Physics Teaching [J], Physics Teacher, 2012, 12 (03): 18-19;

[5] Zhang Baoqing, Exploration for Teaching Methods of Physics History under New Curriculum Standard [J], Physics Teaching, 2012, 09 (01): 28-29;

[6] Chen Minhua, Introduction to German Karlsruhe Physics Course [J], Physics Teacher, 2008, 08 (05): 39-40;

[7] Ruan Shenglei, Significance of Physical Theory Structure for Physics Teaching [D], Shanghai Normal University, 2013. 